\title{
Research Square \\ Grey Wolf Optimization Based Energy Efficiency Management System for Wireless Sensor Networks
}

S. Jaya Pratha ( $\sim$ jayapratha109@gmail.com )

Annamalai University https://orcid.org/0000-0001-6648-590X

\section{Asanambigai}

Annamalai University

\section{S.R. Mugunthan}

RVS College of Engineering and Technology

\section{Research Article}

Keywords: Sensor node, Energy Efficiency, Meta-heuristic Optimization, Grey wolf Optimization, Game theory

Posted Date: July 12th, 2021

DOI: https://doi.org/10.21203/rs.3.rs-397273/v1

License: (c) (i) This work is licensed under a Creative Commons Attribution 4.0 International License. Read Full License 


\title{
Grey Wolf Optimization based Energy Efficiency Management System for Wireless Sensor Networks
}

\author{
S. Jaya Pratha ${ }^{1}$ Dr.V.Asanambigai ${ }^{2}$ Dr.S.R.Mugunthan ${ }^{3}$
}

\begin{abstract}
Wireless Sensor Networks (WSN) is the fundamental technology for the Internet of Things (IoT). It is a network formed from several sensor nodes to sense the changes in the environment. The nodes are battery powered that performs sensing and transmission of information to other nodes in the network. Thus, the energy of the sensor node plays a crucial role in WSN. Thus, intelligent models are anticipated to solve the network problems by optimizing or minimizing the mechanism inorder to improve the energy efficiency. In this paper, a combined meta-heuristic approach called Grey Wolf Optimization based Game theoretical Approach (GWOGA) is proposed that helps for clustering to find the best solutions for selection of aggregation points and this optimal selection of aggregation points lead the nodes to maximize its battery/lifetime. Experimental and simulation analysis shows that the GWOGA outperforms the existing models and retains the lifetime of the network.
\end{abstract}

Keywords: Sensor node, Energy Efficiency, Meta-heuristic Optimization, Grey wolf Optimization, Game theory

\section{Introduction}

Wireless Sensor Networks (WSNs) is one of the emerging technologies of wireless ad-hoc networks used in our day-to-day lives that collect process and communicate data collected from the physical environment. Thereby WSN connects the physical world to the digital world with cost efficient and faster communication. Nowadays variety of applications like transportation, patient monitoring in health care systems, military, industrial surveillance, electrical appliances in home, etc. involves WSN as it uses small, low cost and intelligent sensors for transmitting control messages and instructions from the physical environment to the external Base Station (BS) [3,22]. This intelligence changes the world a way ahead and all these applications of WSN involve the sensor nodes.

The sensor node for example sensing the humidity, temperature for weather, manages a sensing work. Moreover, these sensor nodes can be left without watching as these nodes does the task of sensing, it sends it to the base station [8]. As sensing nodes are small, they are deployed all over the region to monitor the characteristic changes occurs in the particular area. The sensors which operate on batteries needs to active for a prolonged duration as it transmits emergency messages to the BS which is located in the particular sensing area [9]. Thus, the network lifetime depends on the operating sensors and the lifetime of the network needs to be prolonged when the battery fluctuates. In WSN, the network stability is very important for managing the network resources efficiently. 
The sensors involved in WSN have their unique characteristic features such as processing capability, limited power supply and tasks of communicating and sensing the environment arbitrarily. Among several applications in WSN, the energy wastage occurs mainly due to transmitting data to and from the environment. Thus, the sensing area contains sensors with nonrechargeable batteries. So, once the power of the sensors is running out, then they need to be replaced with new ones. Therefore, the scheme of energy efficient routing with minimal power consumption is the major challenges in WSN [14]. Nowadays most of the day-to-day applications uses WSN, thus researchers are moving towards finding an optimal and novel clustering algorithm to improve the performance of the existing algorithm by designing meta-heuristic techniques. The existing clustering algorithms are classified into classical clustering methods and meta-heuristic techniques. There are five main categories in classical clustering algorithms such as partitional clustering, grid-based clustering, density-based clustering, model-based clustering and hierarchical clustering [4, 25].

Most of the recent research optimization techniques in WSN such as Ant Colony Optimization, Particle Swarm Optimization and Fuzzy logic-based algorithms have been proposed to extend the lifetime of the network $[1,13]$. Hierarchical routing protocols such as clustering are mainly used to improve the network lifetime. In clustering, the entire region is separated into different cluster sensing areas. Then, for a particular sensing area a single cluster head is chosen and it transmits the information to the centralized BS $[15,16]$. A hierarchical routing mechanism is performed between the cluster member and the cluster head. Here the cluster head selection plays an important role because the lifetime and stability of the network depends upon the cluster head $[6,20,21]$.

Nowadays researchers concentrate towards meta-heuristic algorithms to overcome the issues faced by the clustering techniques. With the help of the novel meta-heuristic algorithms, clustering problems are solved in an optimal and efficient manner with less computational time. The results from the algorithms proposed in [13] are not satisfactory, thus many improvements are needed to solve the clustering problem with the help of meta-heuristic approaches $[5,24]$.

In this paper, we proposed a meta-heuristic approach called Grey Wolf Optimization (GWO) for performing the clustering algorithm in WSN. Among other swarm optimization algorithms, GWO is the intelligent technique as it involves few parameters for computation and no more derivation is involved for initial search operation. Grey wolves are placed on the top of the food chain. Their characteristic such as attacking, encircling and tracking is compared to the areas in WSN. The major contribution of our paper is to find an optimal clustering approach using GWO. GWO is based on the behaviour of the wolves for optimization algorithms. As GWO is simple, efficient, flexible to use and it is one of the global optimization algorithms and it gives better results with accuracy. By implementing GWO in feature subset selection algorithm, the algorithm proves that it shows an improved performance than the other particle swarm optimization algorithms. 
The rest of this paper is summarized as follows. The literature review is described in Section 2. Section 3 provides the problem description and the proposed algorithm. Section 4 discusses the performance and result analysis with comparison study. In conclusion, sections 5 are drawn with conclusion and future work.

\section{Related Work}

Nowadays, applications of WSN are growing very rapidly. The sensors are located in order to collect all important information from the sensing environment. The sensing environment needs prolonged data recovery. For that, we need to place low-cost energy efficient sensors. Most of the functions in WSN such as data transmission, process control, environment monitoring, etc. consumes more energy to get the required information from the sensors. Thus, the challenging solution towards WSN is to maintain the network lifetime with limited battery powered sensors. Many researchers are working towards energy efficient algorithms to prolong and retain the network lifetime. Clustering is one of the efficient techniques in which the hierarchical network architecture is separated into different functional layers. Each cluster partition contains the Cluster Head $(\mathrm{CH})$ and it plays an important role to transmit information between the Cluster Members (CMs) [11]. CH aggregates and collects data from different CMs and communicates with the base station using hierarchical routing protocol. The traditional hierarchical routing called LEACH is used to improve the lifetime of the network. The authors in $[18,26]$ proposed an efficient method to overcome the issues of LEACH hierarchical routing protocol by selecting an optimal $\mathrm{CH}$ without considering the node's distance and its energy. Other hierarchical routing algorithms are based on the distance between the BS and the $\mathrm{CH}$, residual energy of the node, etc. [12].

In order to balance the energy requirements of WSN, various schemes are proposed. The authors in [23] proposed a clustering technique to achieve energy efficient routing where a single Cluster Head $(\mathrm{CH})$ is chosen from the cluster. The $\mathrm{CH}$ gets information from all other nodes in the cluster, aggregates it and send it to the base station. The $\mathrm{CH}$ receives information in either singlehop or multihop pattern. In singlehop, the sensor node consumes more power when the distance with $\mathrm{CH}$ is more. But in multihop, when the distance with $\mathrm{CH}$ is less, then the nodes consume more power because of relaying through different nodes. Here, again the $\mathrm{CH}$ selection plays a vital role. In [23], the authors proposed a QoS based $\mathrm{CH}$ selection technique to minimize the energy consumption and load of the entire network. For a distributed multihop environment, the authorhad chosen the $\mathrm{CH}$ from the cluster by their proposed Deterministic Stable Election Protocol (D-SEP). For heterogeneous network, the authors in [27] proposed a Balanced EnergyEfficient Grouping (BEEG) protocol where each group having one $\mathrm{CH}$ associated with them. The main disadvantage of this approach is that it takes more time and consumes more energy for each user request.

The energy efficient Low-Energy Adaptive Clustering Hierarchy (LEACH) algorithm is proposed in [10], where the each and every node can be elected as a $\mathrm{CH}$ after some particular interval of time. Thus, this technique ensures the active participation of each and every node in the cluster. Thus, the energy consumption is distributed among all the nodes in the cluster, But in 
centralized LEACH approach, the decision of $\mathrm{CH}$ selection is performed in the base station. Thus, the energy consumption is less in LEACH-C when compared to LEACH approach. In this way, the base station also collects the information about the nodes in the cluster. Another approach for centralized cluster head selection is Base-station Controlled Dynamic Clustering Protocol (BCDCP) where each cluster is balanced in terms of number of nodes to reduce the overloading problem in the cluster. Here the $\mathrm{CH}$ communicates with the base station to transmit the information from other clusters. Thus, BCDCP outperforms than LEACH-C in terms of processing time and cluster quality [19].

In [20], a markov chain model is devised where the nodes consume more energy when they are in active state and consumes less energy when they are in sleep or inactive state. Distributed Energy-Efficient Clustering (DEEC) is proposed where the $\mathrm{CH}$ gets charged when it diffuses its energy. Adaptive Fidelity Energy-Conserving Algorithm (AFECA) is designed to retain the energy efficiency of the network by switching the sensor node to sleep mode when it doesn't receive any information. But the $\mathrm{CH}$ selection in these algorithms consumes more energy and this clustering process takes more time for data transmission. In [28], the authors proposed the Cluster-based routing algorithm also causes large number of cluster formation which again consumes more energy. WSN for time constrained applications are proposed in [29]. The proposed Data Aggregation Based Routing Scheme (DABRE) avoids the unnecessary transmissions and thus it utilizes the power consumption efficiently and in turn, energy is saved. In addition, one more energy efficiency algorithm was proposed in [30] the energy efficient relay node routing and this algorithm is based on voronoi cells.

Nowadays, researchers concentrate on meta-heuristic algorithms which solves the issues of clustering approach [17]. The authors proposed a simulated annealing algorithm for clustering approach where the clustering process is based on the parameter setting process. Here for parameter setting process vary depends upon the cluster and application requirement. The authors in [10] modelled a K-Means (KM) clustering approach which is widely used for its simpler execution, where the cluster formation is based on mutation operation. But the KM algorithm depends on the initial static state, thus the coverage is not more efficient. A tabu search based heuristic algorithm is designed to combine the cluster formation and diffusion process and it gives better result than simulated annealing and $\mathrm{KM}$ algorithm. Compared to the traditional clustering techniques, the evolving Genetic Algorithms (GA) gives optimal and efficient solution using the steps of mutation, crossover over and selection process.

Among several meta-heuristic algorithms, Ant Colony Optimization (ACO) is modelled. Here the algorithm works similar to the behaviour of ants in which all the ants follow the shortest path from source to destination to find their food source. The performance of ACO is compared with GA and KM algorithm and observes that the ACO algorithm gives better simulation results both in case of time delay and energy efficiency. For data clustering process, an optimization technique called Honeybee Mating techniques is proposed where the quality of cluster is improved [7]. In Particle Swarm Optimization (PSO) algorithm, the clustering is performed based on the 
flocking behaviour of the bird. In order to get better coverage rate and less computation complexity, the KM algorithm is combined with PSO and the simulation results are compared. But PSO algorithms fails when there is overlapping of points in the dataset. Another optimization algorithm called Artificial Bee Colony ( $\mathrm{ABC}$ ) algorithm is proposed where the data clustering is improved based on the forging behaviour of bees. GWO is another meta-heuristic algorithm [2], which shows better performance than PSO and other genetic algorithms. GWO works based on the hunting behaviour of grey wolves.

\section{Proposed Work}

\section{1. Problem Description}

In order to design energy efficient protocol for wireless sensor networks, first the nodes in the sensing area is identified and common cluster grouping is done between them. This clustering is performed based on our proposed meta-heuristic Grey Wolf Optimization (GWO) algorithm. The proposed GWO based clustering algorithm is modelled for Wireless Sensor network to make energy efficient clustering between the sensor nodes. The behaviour of grey wolf is depicted into mathematical models. Thus, this mathematical model plays a vital role in efficient and optimized cluster formation. In each cluster, the cluster head is chosen with the help of game theory algorithm based on its energy and distance from the base station. The end results are depicted graphically and comparison is done in the performance analysis section.

\subsection{Grey Wolf Optimization based Clustering (GWO-C)}

GWO algorithm mimics the behaviour of grey wolves such as hunting, searching, encircling and attacking the prey. Grey wolves are the predators in the top of the food chain and they live in a group on an average of 5-12. Figure. 1 explains the categorization of wolves; the top ones in the pyramid are called alpha wolves. The hierarchy consists of 4 types of wolf groups such as alpha( $\alpha)$, beta $(\beta)$, gamma $(\delta)$, and omega $(\omega)$ wolves. The important hunting decisions are taken by $\alpha$ wolves with the help of consultants given by $\beta$ wolves. The $\beta$ are replaced by $\alpha$ when $\alpha$ becomes old. The leaf of the tree is $\omega$ wolves and they are controlled by $\delta$ wolves. The $\delta$ wolves provides information to the $\alpha$ and $\beta$ wolves.

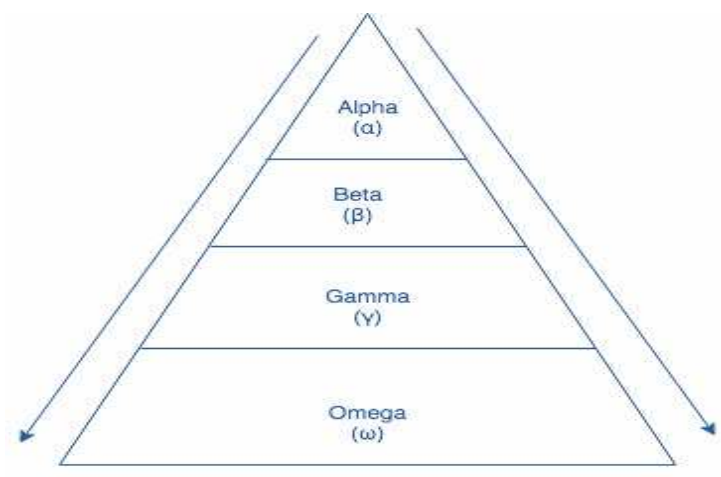

Figure 1 Hierarchy of Grey Wolves 
The social behaviour of grey wolves helps in efficient solving of problems and finds an optimal solution. The wolves' characteristics such as hunting, deciding, searching and tracking are linked with the communication network and for the wireless sensor networks as shown in the equation 1; it deals with the efficient clustering formation. In our proposed GWO-C algorithm, the hierarchy of grey wolves are modelled mathematically to perform efficient clustering.

$\underline{X}(\mathrm{i}+1)=\underline{X} \underline{\mathrm{p}}_{\mathrm{p}}(\mathrm{i})-\underline{Y} \cdot \underline{Z}$

$\underline{Y}=2 \mathrm{a} \cdot \mathrm{r}_{1}-\mathrm{a}$

$\underline{Z}=\left|\mathrm{K} \cdot \underline{X}_{\mathrm{p}}(\mathrm{i})-\underline{X}(\mathrm{i})\right|$

$\mathrm{K}=2 . \mathrm{r}_{2}$

Where $\mathrm{r} 1$ and $\mathrm{r} 2$ are random vectors

$\underline{X}$ is the grey wolf position and $\underline{X p}$ is the hunting position

Compared with the traditional models, meta heuristic models are proposed to improve the performance of the overall network. To utilize less computation overhead and to provide optimal solutions we go for grey wolf optimization. Algorithm 1 describes GWO-C and is easy to implement and efficient optimization algorithm that is based on the behaviour of grey wolves. The social behaviour of grey wolves has three major steps involved such as hunting for the prey, encircling for the prey and attacking to the prey where the cluster formation is also optimized with the three best solutions. The ability of GWO-C is strengthened and the architecture of GWO-C is as shown in the Figure 2.

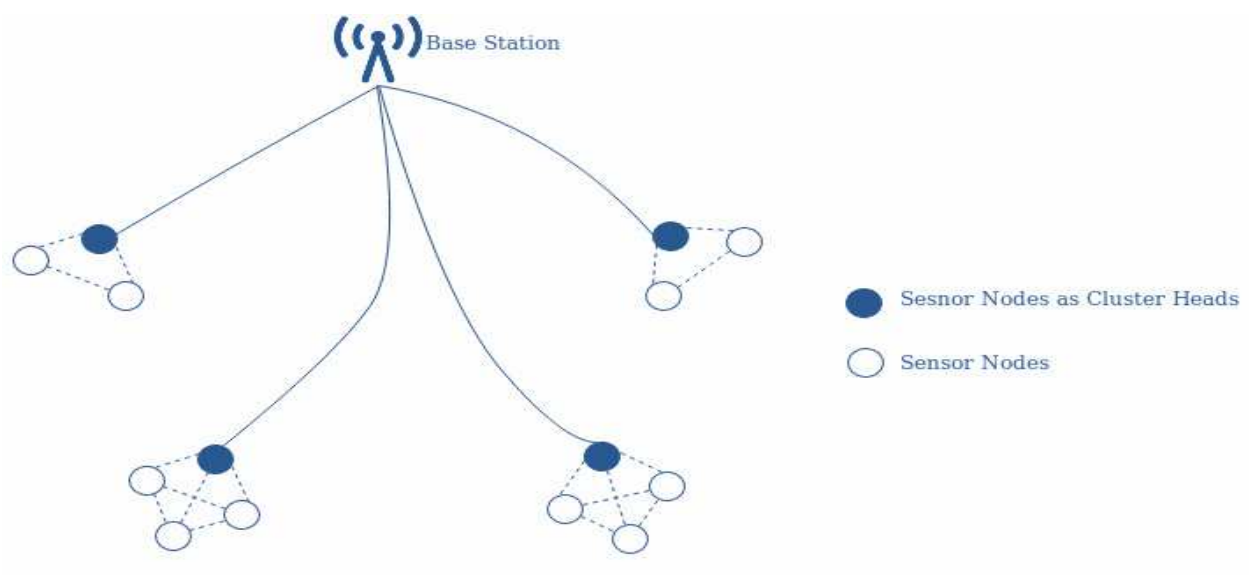

Figure 2 GWO-C Architecture 


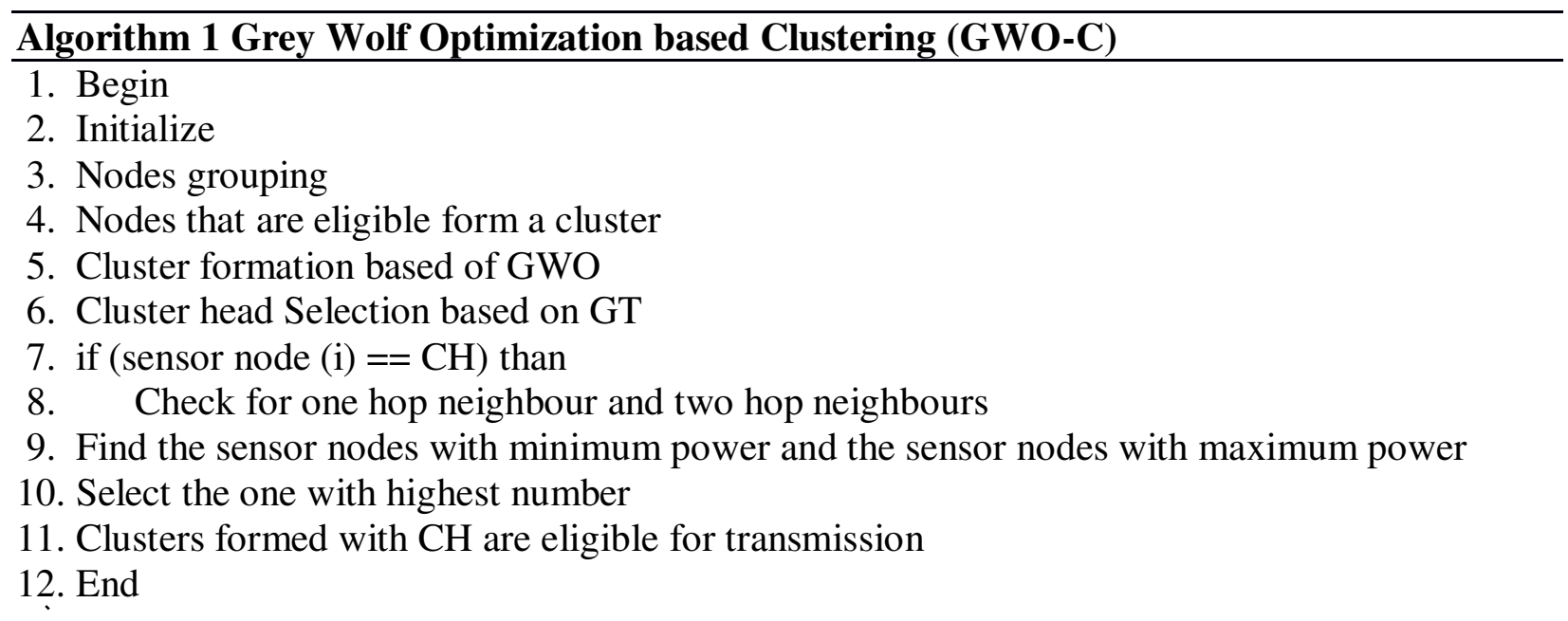

Clustering is a process in which all the sensor nodes are categorized into number of clusters based on the sensor node and application criteria. Each clustering follows some unique properties. The clusters should have at least more than one sensor node. Based on the characteristics such as maximum transmission distance it is categorized as alpha, beta, gamma and omega clusters. Cluster formation based on Euclidean distance, geographical co-ordinates, neighbour discovers and hop counts.

Consider the sensors $(1 \ldots . \mathrm{N})$. Assume $\mathrm{N}$ be 100 , the spatial co-ordinates $\left(\mathrm{N}_{\mathrm{x} 1, \mathrm{y} 1}\right)$ to $\left(\mathrm{N}_{\mathrm{xj}, \mathrm{yj}}\right)$ than the Euclidean formula is given by

$$
c\left(x_{i}, y_{i}\right)=\sqrt{\left(x_{i}-x_{i-1}\right)^{2}+\left(y_{i}-y_{i-1}\right)^{2}}
$$

The sensor nodes senses, transmits, receives and changes from sleep to active state and on all these states each time an amount of energy is consumed. The refreshing operation is initiated to move from sleep to active state. Considering the operations of the sensor nodes and it follows a probability distribution with a rate $\lambda$,

Considering a contract time $t$, the probability is given by

$$
\mathrm{P}\left(\mathrm{T}_{\mathrm{s}}\right)=\int_{t=0}^{t} e^{-\lambda t} \mathrm{dt}
$$

To extend the battery life of each individual sensor node we form clustering using grey wolf optimization. In addition, based on the cluster formation we elect efficient $\mathrm{CH}$ based on the player's strategy and pay off. Thus, the dynamic reconfiguration makes sure that the elected $\mathrm{CH}$ is efficient and the network is balanced with the power usage with no sensor resources are battery drained. The sensor nodes that are involved for the complete application which can co-ordinate among other sensor nodes are chosen in alpha groups. Base station will communicate with the sensor node and determines the location, battery life of each sensor nodes. The information for the alpha groups is given by gamma groups. 
In each cluster, the $\mathrm{CH}$ selection plays an important role in GWO-C algorithm. This $\mathrm{CH}$ selection is based on Game Theory approach. The $\mathrm{CH}$ master ship is rotated with other nodes and the base station checks the threshold distance value as it considered, as SENS $_{\text {dis }}$ is calculated using Euclidean distance as shown in equation 2. SENS loc is calculated using the spatial co-ordinates of the sensor nodes and base station.

Clustering game, a game theoretic approach is played among the sensor nodes to choose a $\mathrm{CH}$. The duty of the $\mathrm{CH}$ is to gather information of all sensor nodes that are present in the cluster. In addition, the $\mathrm{CH}$ needs to efficiently aggregate the data to the base station. A game with set of $\mathrm{N}$ players participating in the network. The strategy ( $\mathrm{S}$ ) among the players is considered as $\left\{\mathrm{X}_{\mathrm{i}}\right\}$ as follows.

$$
\begin{gathered}
\mathrm{N}=\{1,2,3 \ldots \mathrm{n}\} \\
\mathrm{S}=\left\{\mathrm{X}_{\mathrm{i}}\right\} \mathrm{F}_{\mathrm{i}}=: \mathrm{X}=\mathrm{X}_{\mathrm{i}} \rightarrow \mathrm{R} \\
\mathrm{U}=\{\mathrm{A}, \mathrm{A}, \mathrm{B}\} / / \mathrm{A}-\text { not declaring CH, B-declaring } \mathrm{CH} \\
\mathrm{CH}-\mathrm{GT}=\{\mathrm{N}, \mathrm{S}, \mathrm{U}\}
\end{gathered}
$$

A node in the cluster set is allowed to decide to be a $\mathrm{CH}$ or not and this master ship is rotated among the players in the cluster set (sensor nodes of the set). The strategy is a normal form (R), and the strategy is going to be either B for CHs or A other than the CHs. In addition, if none of the players is eligible with $\mathrm{CH}$ then the utility payoff would be zero and it does not attain Nash equilibrium. Thus, there should be atleast one sensor node to be declared $\mathrm{CH}$ and the payoff is set to PU. Thus, the total cost is the total pay off and for each $\mathrm{CH}$ and $\mathrm{PU}_{\mathrm{i}}$ is reduced from the total payoff.

Assume for a 2-node set the possible strategies are $\{A, B\},\{A, A\},\{B, A\},\{B, B\}$. Thus, if the set belongs to $\{\mathrm{A}, \mathrm{B}\}$ or $\{\mathrm{B}, \mathrm{A}\}$ than the cluster attains Nash equilibrium otherwise the set $\{A, A\}$ or $\{B, B\}$ does not attain the Nash equilibrium state. Utility is the probability of atleast one of the nodes is denoted as $\mathrm{CH}$ using master ship strategy and no one else in the cluster is declared as $\mathrm{CH}$ and it is equated as follows

$$
\mathrm{U}=\mathrm{PU}(1-\mathrm{Pr} \text { (no one else declaring CH-GT)) }
$$

The $\mathrm{CH}$ node energy used for the transmission of $\mathrm{M}$ packets and receiving of $\mathrm{K}$ packets is given by,

$$
\begin{aligned}
& \mathrm{CH}-\mathrm{GT}=\mathrm{M} \text { bits (TE) } \\
& \mathrm{CH}-\mathrm{GT}=\mathrm{K} \text { bits (RE) }
\end{aligned}
$$

Thus, the $\mathrm{E}_{\mathrm{Agg}}$ energy aggregated for both transmission and reception is given by,

$\mathrm{E}_{\mathrm{Agg}}=\mathrm{M}$ bits $(\mathrm{TE})+\mathrm{K}$ bits $(\mathrm{RE})$

If $\mathrm{E}_{\mathrm{Agg}} \geq \mathrm{T}$ than the cluster master ship is rotated. 
Rules for Cluster master-ship

1. A sensor node in a cluster can be declared any number of times the master ship provided the remaining energy of the node is greater than the threshold energy.

2. A sensor node in a cluster can also never be declared cluster master ship due its low energy.

3. Based on these rules with the game approach the maximum nodes lifetime is achieved.

\section{Performance and Result Analysis}

In this section the results related with energy and network lifetime of the sensor nodes obtained using the proposed algorithm are discussed. The performance of the wireless sensor networks using game theory with grey wolf optimization is simulated in MATLAB and the results are compared with the other proposed Energy Efficient Relay Node Routing (ERNR) Algorithms. The thorough simulation tests with the simulated sensor nodes show that the proposed GAGWO algorithm gives energy efficiency with higher throughput. The results show that the proposed GAGWO consumes only half the energy than the existing algorithms.

Conferring to the result, the parameters considered for minimizing the energy consumption is discussed in the Table I.

Table I Simulation Parameters

\begin{tabular}{|l|l|}
\hline Parameters & Values \\
\hline Number of Nodes & 200 \\
\hline Simulation Network size & $100 * 100$ \\
\hline Base Station Initial Position & $(50,50)$ \\
\hline Maximum allowed rounds & 1500 \\
\hline Channel bandwidth & $1 \mathrm{Mbps}$ \\
\hline Proposed Approach & GAGWO \\
\hline GAGWO Compared With & ERNR \\
\hline Transmitting Energy & $50 \mathrm{~nJ} / \mathrm{bit}$ \\
\hline Receiving Energy & $30 \mathrm{~nJ} / \mathrm{bit}$ \\
\hline Sensing Energy & $10 \mathrm{~nJ} / \mathrm{bit}$ \\
\hline Message Transmission Radius & $(25,50,75)$ \\
\hline Load & $1500 \mathrm{~Kb}$ \\
\hline Sensor Radius & 40 \\
\hline Packet Size & $100 \mathrm{bytes}$ \\
\hline CH probability & 0.1 \\
\hline Initial Energy & $0.5 \mathrm{~J}$ \\
\hline
\end{tabular}




\subsection{Comparative Analysis}

A comparison between GAGWO and ERNR algorithms are discussed in the following:

It is found that the proposed GAGWO algorithm was about $15 \%$ better than the ERNR algorithm. The values obtained from the ERNR and GAGWO are observed and plotted as shown in the Figure 3. However, the errors are expected due to the linearity assumption of the algorithm. Thus, there is a reduction in the error value than the existing work. As a result, it adds an advantage to the energy usage consumption. In addition, when number of sensor nodes increases, then the ERNR error value prediction grows linearly wherein the proposed GAGWO approach, the error rate is lesser than the ERNR algorithm.

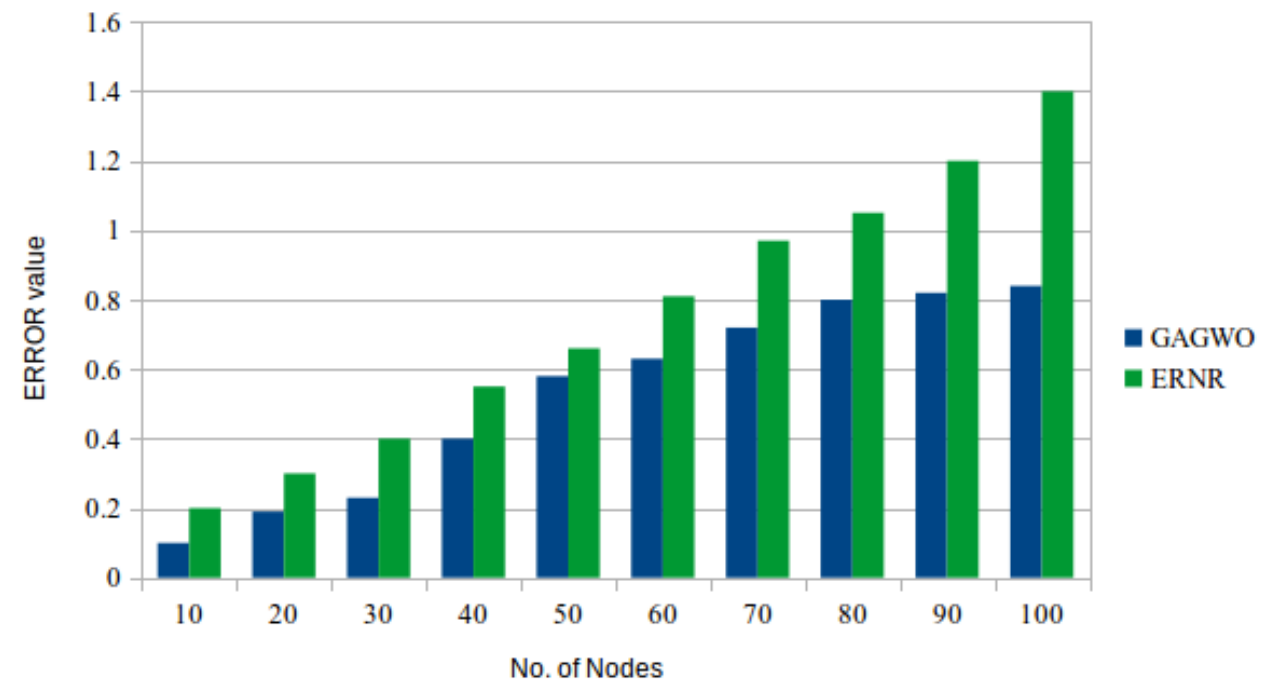

Figure 3. Comparison of ERROR Values

The simulation was run around 80 times and fed with different message transmission radius as 25,50 and $75 \mathrm{~m}$ with 150 to 200 sensor nodes; the transmission radius is set before the start of the simulation and then evaluated, the efficiency achieved on an average is greater than $75 \%$. The efficiency is evaluated with the energy consumption based on average distance among nodes, average number of neighbours and average number of clusters. The transmission delay observed is set to maximum of 12 seconds. In addition, the efficiency is plotted as in Figure 4. 


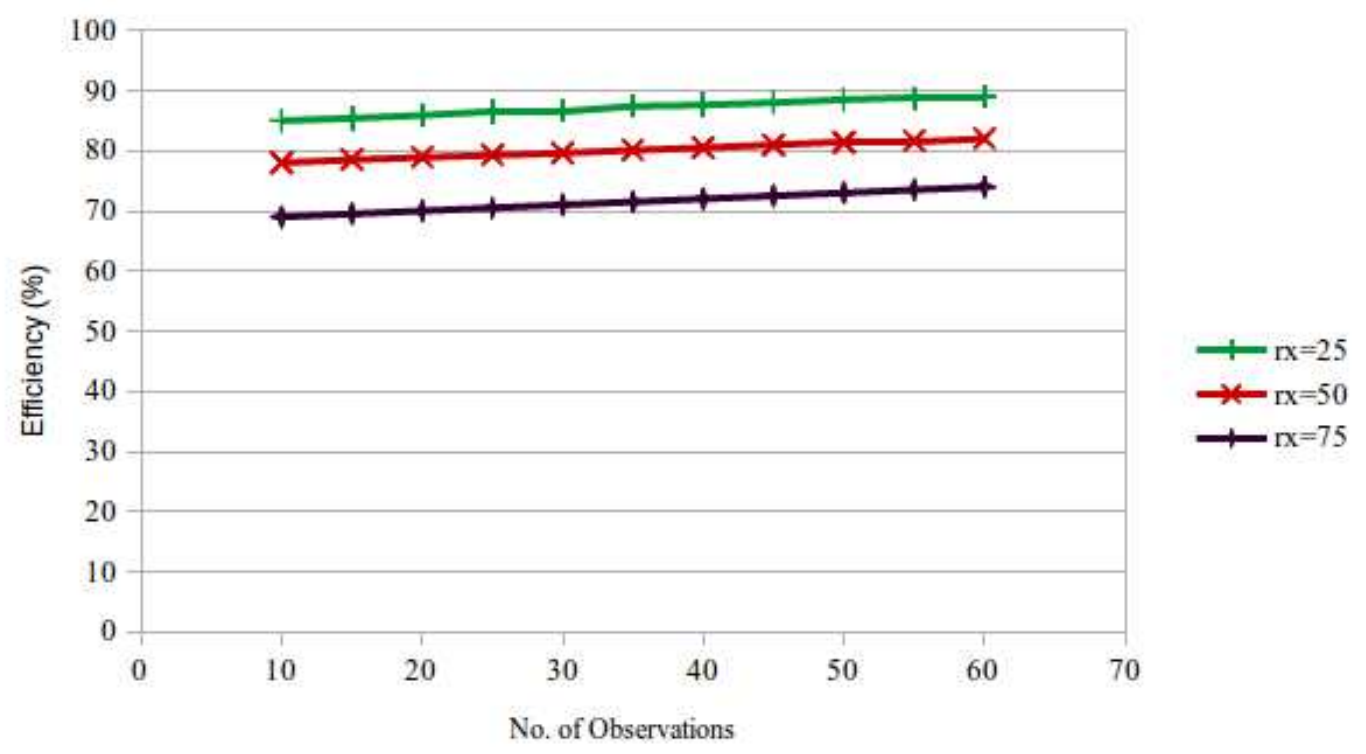

Figure 4. Efficiency Vs No of Observations

The efficiency is calculated for GAGWO and ERNR based on the network load to a maximum of $1000 \mathrm{~Kb}$. The GAGWO performs better than the ERNR and it is shown in Figure 5.

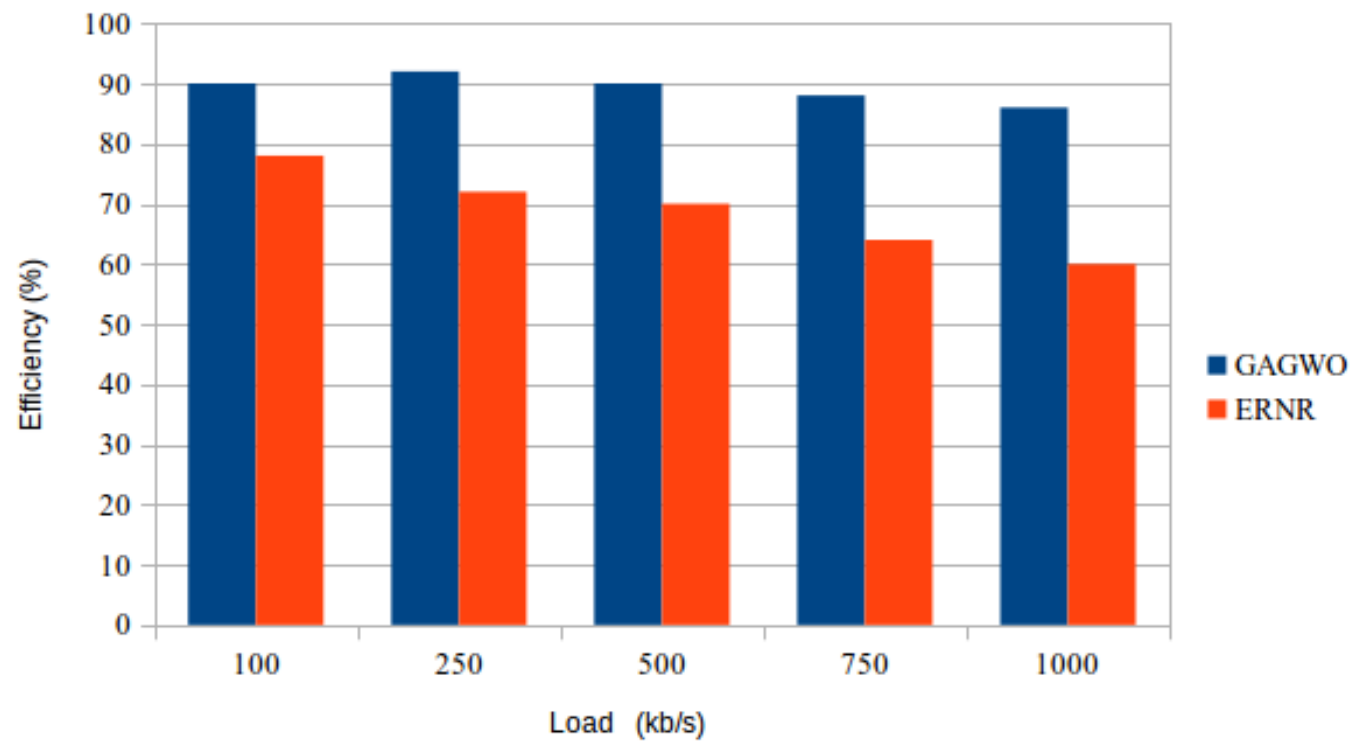

Figure 5. Efficiency Vs Load Analysis

The reduction in energy is pictorially represented in Figure 6. The graph explains that on an average of 1600 observations were taken and at around 1500 observation the energy of the sensor nodes is completely deprived and compared to the ERNR algorithm where the sensor nodes energy is deprived after 1000 observations, thus the total efficiency is $50 \%$ greater than the ERNR. 


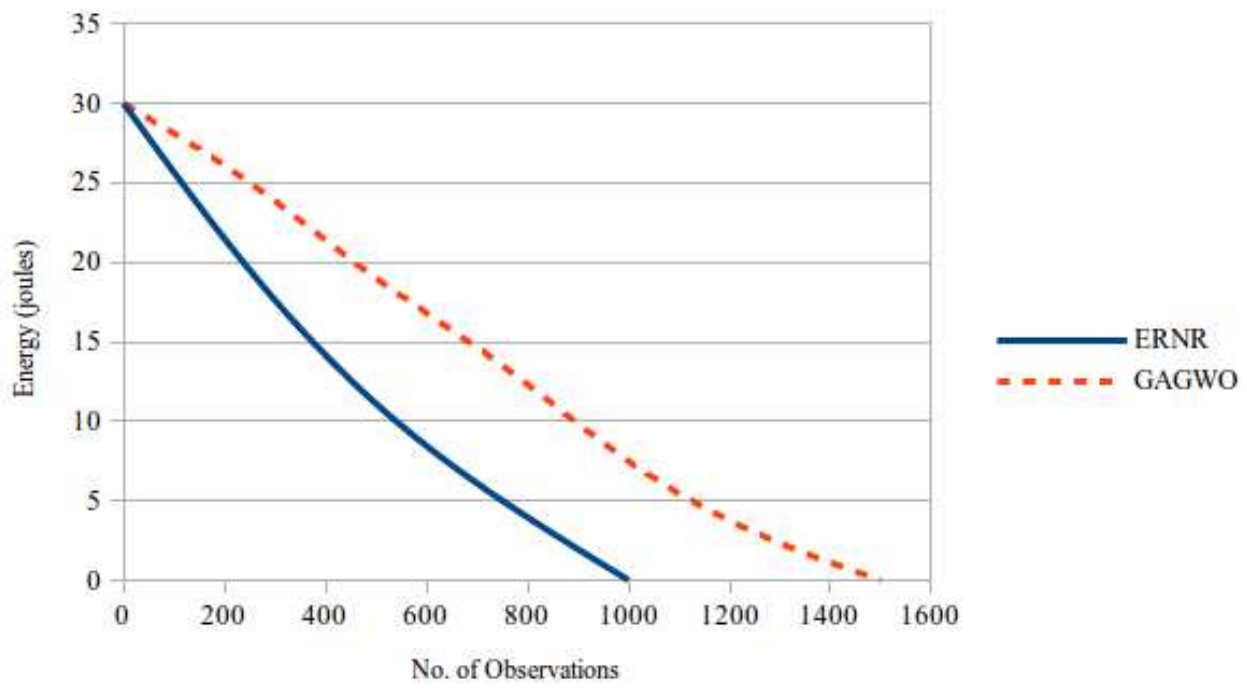

Figure 6. Energy Vs No of Observations

With respect to sensor nodes the last sensor nodes get deprived in 1000 rounds while considering ERNR algorithm, wherein the proposed GAGWO the last sensor nodes withstands on an average of 1500 to 1600 rounds. The sensor nodes observation is clearly depicted in Figure 7.

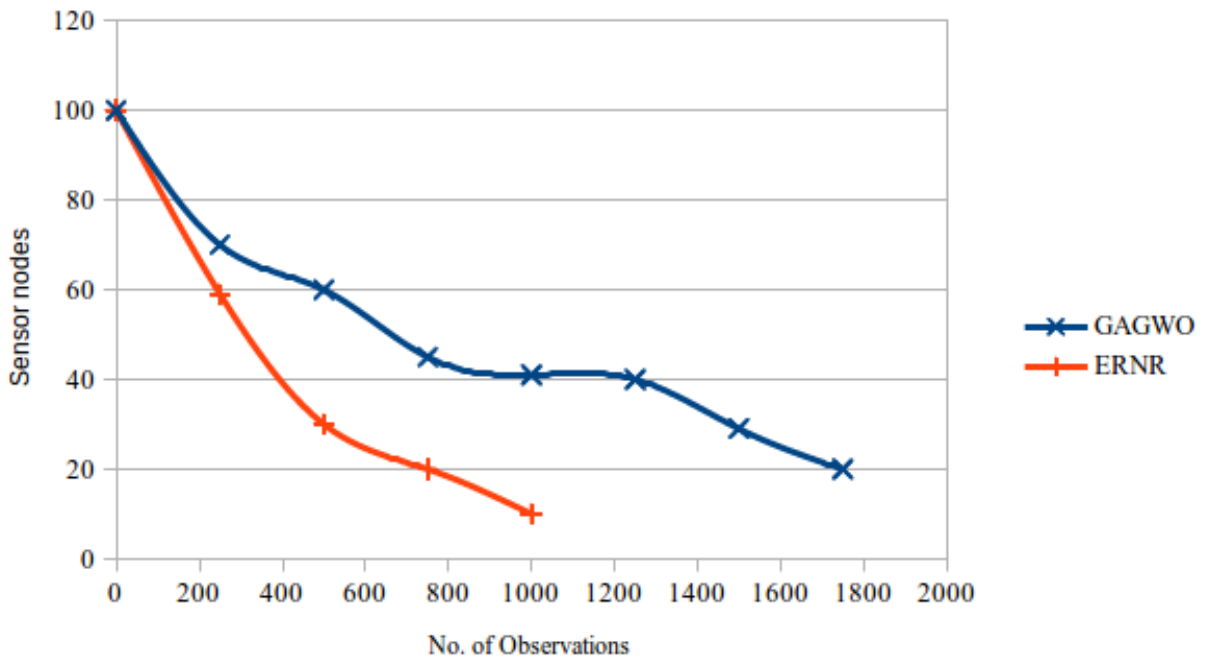

Figure 7. No. of Sensor Nodes Vs No of Observations

\section{Conclusion and Future Work}

In this paper, game theory based grey-wolf optimization was proposed which is a clusterbased approach and this approach shows significance in the energy usage efficiently. To conclude that one of the major factors that affects energy is the transmission distance. Thus, the clusters among sensor nodes are formed based on base station distance and intra distance among sensors. 
On an overall, the network lifetime is maximized. However, the energy is efficiently utilized by the proposed algorithm can be increased if we adapt machine learning and intelligence techniques.

\section{References}

1. Z. Wang, W. Xu, J. Yang and J. Peng, "A Game Theoretic Approach for Resource Allocation Based on Ant Colony Optimization in Emergency Management," International Conference on Information Engineering and Computer Science, Wuhan, pp. 1-4, 2009.

2. D. Jitkongchuen, P. Phaidang and P. Pongtawevirat, "Grey wolf optimization algorithm with invasion-based migration operation," IEEE/ACIS 15th International Conference on Computer and Information Science (ICIS), Okayama, pp. 1-5, 2016.

3. Diwan, P. and Khan, M.R., "Energy Efficient Communication for WSNs using Grey-Wolf Optimization Algorithm," International Journal Of Engineering And Computer Science, vol. 5, no. 12, 2016.

4. Yang, G., "Game Theory-Inspired Evolutionary Algorithm for Global Optimization," Algorithms, vol. 10, no. 4, pp. 111, 2017.

5. AlSkaif, T., Zapata, M.G. and Bellalta, B., "Game theory for energy efficiency in wireless sensor networks: Latest trends," Journal of Network and Computer Applications, vol. 54, pp. 33-61, 2015.

6. A. Attiah, M. Chatterjee and C. C. Zou, "A Game Theoretic Approach for Energy-Efficient Clustering in Wireless Sensor Networks," IEEE Wireless Communications and Networking Conference (WCNC), San Francisco, CA, pp. 1-6, 2017.

7. Rajakumar, R., Amudhavel, J., Dhavachelvan, P. and Vengattaraman, T., "GWO-LPWSN: Grey wolf optimization algorithm for node localization problem in wireless sensor networks," Journal of Computer Networks and Communications, 2017.

8. B. Sreevidya and M. Rajesh, "Enhanced energy optimized cluster based on demand routing protocol for wireless sensor networks," International Conference on Advances in Computing, Communications and Informatics (ICACCI), Udupi, pp. 2016-2019, 2017.

9. Al-Karaki, Jamal N., RazaUl-Mustafa, and Ahmed E. Kamal. "Data aggregation in wireless sensor networks-exact and approximate algorithms," High Performance Switching and Routing (HPSR) IEEE Workshop, pp. 241-245. 2004.

10. N. A. Al-Aboody and H. S. Al-Raweshidy, "Grey wolf optimization-based energy-efficient routing protocol for heterogeneous wireless sensor networks," 4th International Symposium on Computational and Business Intelligence (ISCBI), Olten, pp. 101-107, 2016.

11. Priyadarshini, R.R. and Sivakumar, N., "Cluster head selection based on Minimum Connected Dominating Set and Bi-Partite inspired methodology for energy conservation in WSNs," Journal of King Saud University-Computer and Information Sciences, 2018.

12. Rault, T., Bouabdallah, A. and Challal, Y., "Energy efficiency in wireless sensor networks: A top-down survey," Computer Networks, vol. 67, pp. 104-122, 2014.

13. Yadav, A., Kumar, S. and Vijendra, S., "Network Life Time Analysis of WSNs Using Particle Swarm Optimization," Procedia Computer Science, vol. 132, pp. 805-815, 2018. 
14. Le, T.N., Pegatoquet, A., Le Huy, T., Lizzi, L. and Ferrero, F., "Improving energy efficiency of mobile WSN using reconfigurable directional antennas," IEEE Communications Letters, vol. 20, no. 6, pp. 1243-1246, 2016.

15. Padilla, P., Camacho, J., Maciá-Fernández, G., Díaz-Verdejo, J.E., Garcia-Teodoro, P. and Gómez-Calero, C., "On the influence of the propagation channel in the performance of energyefficient geographic routing algorithms for wireless sensor networks (WSN)," Wireless personal communications, vol. 70, no. 1, pp. 15-38, 2013.

16. Pandey, O.J. and Hegde, R.M., "Low-Latency and Energy-Balanced Data Transmission over Cognitive Small World WSN," IEEE Transactions on Vehicular Technology, 2018.

17. Kumar, V., Chhabra, J.K. and Kumar, D., "Grey wolf algorithm-based clustering technique," Journal of Intelligent Systems, vol. 26, no.1, pp. 153-168, 2017.

18. Ozger, M. and Akan, O.B., "Event-driven spectrum-aware clustering in cognitive radio sensor networks," IEEE INFOCOM, pp. 1483-1491, Apr. 2013.

19. Koltsidas, G. and Pavlidou, F.N., "A game theoretical approach to clustering of ad-hoc and sensor networks," Telecommunication Systems, vol. 47, no. 1-2, pp. 81-93, 2011.

20. Mishra, M., Panigrahi, C.R., Sarkar, J.L. and Pati, B., "Gecsa: A game theory based energy efficient cluster-head selection approach in wireless sensor networks," International Conference on Man and Machine Interfacing (MAMI), pp. 1-5, Dec. 2015.

21. Heloulou, I., Radjef, M.S. and Kechadi, M.T., "Automatic multi-objective clustering based on game theory," Expert Systems with Applications, vol. 67, pp. 32-48, 2017.

22. Karthikeyan, V., Vinod, A. and Jeyakumar, P., "An Energy Efficient Neighbour Node Discovery Method for Wireless Sensor Networks," arXiv preprint arXiv:1402.3655, 2014.

23. Pati, B., Sarkar, J.L. and Panigrahi, C.R., "ECS: an energy-efficient approach to select clusterhead in wireless sensor networks," Arabian Journal for Science and Engineering, vol. 42, no. 2, pp. 669-676, 2017.

24. Mirjalili, S., Mirjalili, S.M. and Lewis, A., "Grey wolf optimizer," Advances in engineering software, vol. 69, pp. 46-61, 2014.

25. V. Kumar, J. K. Chhabra and D. Kumar, "Variance based harmony search algorithm for unimodal and multimodal optimization problems with application to clustering," Cybernet. Systems, vol. 5, pp. 486-511, 2014.

26. S. Tyagi, S. Gupta, S. Tanwar, and N. Kumar, "Ehe-leach: Enhanced heterogeneous leach protocol for lifetime enhancement of wireless sns," International Conference on Advances in Computing, Communications and Informatics (ICACCI), Aug 2013, pp. 1485-1490.

27. Liaw, J.; Chang, L.; Chu, H, "Improving lifetime in heterogeneous wireless sensor networks with the energy-efficient grouping protocol," International Journal of Innovative Computing Information and Control, vol. 8, no. 9, pp. 6037-6047, 2012.

28. G. Chen, C. Li, M. Ye, and J. Wu, "An Unequal Cluster-Based Routing Protocol in Wireless Sensor Network,” Wireless Networks, pp. 193-207, 2007. 
29. J. Martinsahayaraj,N. Dhasarathan,"A Novel Data Aggregation Scheme for Energy Efficient Wireless Sensor Networks ",International Journal of Applied Science Engineering \& Management, Vol 2, Issue 12,Issn: 2454-9940.

30. J.Martinsahayaraj, and J. M. Ganaseakar."Relay node selection with energy efficient routing using hidden Markov model in wireless sensor networks." International Journal of Networking and Virtual Organisations 19, no. 2-4 (2018): 176-186. 